\title{
Autotransplantation de canine maxillaire incluse: à propos d'un cas clinique.
}

\section{Tondelier C1, Champagne JB22, Hamada M³, Leverd C4, Devisse $\mathbf{M}^{5}$}

1. Interne en Chirurgie Orale, UFR Service d'Odontologie, AP-HP - Paris

2. Docteur en Chirurgie Dentaire, UFR Service d'Odontologie, UF d'Implantologie - Service d'Odontologie, CHRU de Lille

3. Spécialiste en Médecine Bucco-Dentaire, Pratique libérale - France

4. Interne en Médecine Bucco-Dentaire, UFR Service d'Odontologie, UF Odontologie - Service d'Odontologie, CHRU de Lille

5. Spécialiste en Chirurgie Orale, UFR Service d'Odontologie, UF Odontologie - Service d'Odontologie, CHRU de Lille

\section{Introduction}

L'autotranplantation est le transfert d'une dent, d'une alvéole à une autre préalablement préparée, chez le même individu. Elle peut être une solution de prise en charge de la canine maxillaire incluse, qui concerne 0,9 à $2 \%$ de la population.

\section{Observation}

Une patiente de 41 ans a consulté dans le service d'Odontologie du Centre Hospitalier Régional Universitaire de Lille face à la persistance des canines temporaires maxillaires devenues mobiles. Les examens clinique et radiologique mettaient en évidence une denture mixte, avec inclusion des canines maxillaires permanentes matures (13 et 23) au niveau de la crête alvéolaire et légèrement déportées en palatin, présentant cependant un faible élargissement desmodontal au tiers apical, signe d'ankylose des dents à ce niveau.

Après avoir obtenu un consentement éclairé de la patiente, le plan de traitement retenu a été la mise en place chirurgico-orthodontique de 13 et 23 sur arcade. Une traction orthodontique a donc été tentée sur une période de 14 mois, sans succès, ce qui a conduit à un changement de stratégie thérapeutique. L'autotransplantation des canines maxillaires a alors été entreprise sous anesthésie locale.

Un suivi clinique et radiographique a été entrepris à 7 jours, 20 jours, 1 mois, 3 mois, 7 mois et 10 mois. II a objectivé à 10 mois une ankylose de 13 et 23 , associée à une poche parodontale de $9 \mathrm{~mm}$ en mésio-palatin de 13 , ainsi que des résorptions inflammatoires externes de 13 et inflammatoire interne de 23 associées à une nécrose de ces dents. Suite à ces observations, les traitements endodontiques de 13 et 23 ont été réalisés.

\section{Discussion}

Après échec de la traction ortho-chirurgicale, plusieurs facteurs ont amené à pratiquer l'autotransplantation: les réhabilitations prothétiques ou implantaires sont des solutions intéressantes mais avec leurs inconvénients : perte d'un organe dentaire intacte et coût élevé ; face à l'autotransplantation qui est une solution de temporisation permettant de retarder de quelques années la prise en charge prothétique ou implantaire de l'édentement, voire être un traitement à plus long terme. Des études récentes sur l'autotransplantation de la canine maxillaire incluse montrent un taux de survie (c'est à dire la présence de la canine transplantée sur arcade) allant de 75,3\% à 94,6\% et un taux de succès de $38 \%$ à $93,4 \%$. Les dernières recommandations conseillent la réalisation d'un traitement endodontique à quatorze jours suivant l'autotransplantation des dents matures (à apex fermés). Dans le cas présenté, cette condition a été réalisée avec retard (10 mois), ce qui pourrait expliquer l'apparition des complications pulpaires.

\section{Conclusion}

L'autotransplantation de la canine maxillaire incluse est de nos jours une alternative thérapeutique relativement peu mise en pratique face à l'arsenal thérapeutique déjà existant. Elle doit cependant rester une solution de dernier recours après traitement ortho-chirurgical.

ctondelier@gmail.com

\section{Références}

Gonnissen $\mathrm{H}$ et al. Oral Surg Oral Med Oral Pathol Oral Radiol Endod, Long-term success and survival rates of autogenously transplanted canines. 2010;110(5):570-8.

Arikan F et al. J Periodontol, 5 -year longitudinal study of survival rate and periodontal parameter changes at sites of maxillary canine autotransplantation. 2008;79(4):595-602.

Gault P. Int Orthod Coll ege Eur Orthod, Idiopathic ankylosis-resorption: diagnosis and treatment. 2013;11(3):262-77

(C) The authors, published by EDP Sciences. This is an Open Access article distributed under the terms of the Creative Commons Attribution License 4.0 (http://creativecommons.org/licenses/by/4.0/). 